\title{
Formalization and Verification of RTPS StatefulWriter Module Using CSP
}

\author{
Jiaqi Yin ${ }^{1} \quad$ Huibiao Zhu ${ }^{* 1} \quad$ Yuan Fei $^{* 2}$ Qiwen $\mathrm{Xu}^{3} \quad$ Ruobiao $\mathrm{Wu}^{4}$ \\ ${ }^{1}$ Shanghai Key Laboratory of Trustworthy Computing \\ East China Normal University, Shanghai, China \\ ${ }^{2}$ School of Information, Mechanical and Electrical Engineering \\ Shanghai Normal University, Shanghai, China \\ ${ }^{3}$ Faculty of Science and Technology, University of Macau, China \\ ${ }^{4}$ Huawei Technology Co., Ltd. China
}

\begin{abstract}
The Real Time Publish Subscribe protocol (RTPS), as a Data Distribution Service (DDS) protocol for computer systems, is composed of several modules. We focus on RTPS StatefulWriter Module which has two patterns, reliable pattern and best-effort pattern. As the main module of sending and receiving messages, its security and reliability are of great concern. The formal method can analyze whether it is a highly credible model from the mathematical point of view. Our research pays attention to the reliable pattern. Thus it is of great importance to model and verify whether the pattern is reliable through formal methods. In this paper, we model seven components of the module using Communicating Sequential Processes (CSP). By feeding the models into the model checker Process Analysis Toolkit (PAT), we verify four properties, divergence free, acknowledgement mechanism, data consistency and sequentiality. Consequently, it can be apparently concluded that the pattern of this module is reliable, which totally caters for its specification.
\end{abstract}

Index Terms-RTPS StatefulWriter Module, CSP, PAT, Modeling, Verification

\section{INTRODUCTION}

Data Distribution Service (DDS) is a new generation of distributed real-time communication middleware technology specification developed by Object Management Organization (OMG) based on HLA and CORBA standards. It adopts publish/subscribe architecture, emphasizes data-centric and provides abundant quality of service strategies. The Real Time Publish Subscribe protocol (RTPS), as a Data Distribution Service (DDS) protocol for computer systems, transfers data from publishers to subscribers. StatefulWriter module is one module of RTPS protocol. It has two modes, which are reliable pattern and best-effort pattern. Reliable pattern means the data must be always transferred to subscribers in the specification. Thus, we follow with interest the reliablity of the reliable pattern in the module.

The behavior of the module contains acknowledgement mechanism and heartbeat mechanism. The former guarantees all messages to be received by subsceibers and the latter assures the messages to reach the subscribers. Besides, data consistency and sequentiality need to be ensured in the reliable pattern. Our work is to model and verify the reliable pattern of

*Corresponding authors: hbzhu@ @ei.ecnu.edu.cn (H. Zhu). yuanfei@shnu.edu.cn (Y.Fei). the module. Thus, through formal modeling and verification of StatefulWriter module, the specification can be more precisely modeled and validated, avoiding the ambiguity of natural language description, which has certain guiding significance.

The most related prior work we identifided is a study by Liu et al. [5] that mainly verified the security, activity and priority of DDS in ROS2. In addition, Alaerjan et al. [1] defined the missing functional behavior in DDS dynamic model and the semantics of the new operation using Object Constraint Language (OCL). Some recent research projects [2], [7], [10] have explored analysis and verification of many aspects of DDS, such as real-time performance, security of DDS-based middleware and so on. Our work focuses on the communication dependability of the module's reliable pattern using formal methods.

The remainder of this paper is organized as follows. Section II gives a brief introduction to RTPS StatefulWriter Module, the process algebra CSP and model checker PAT. In Section III, we formalize the seven core components in the module using CSP. We apply the model checker PAT to implement the model and verify four properties in Section IV, including divergence free, acknowledgement mechanism, data consistence and sequentiality. Section V describes the conclusion and future work.

\section{BACKGROUND}

This section detailedly describes the flows of the module which are used in the next section and briefly introduces the process algebra CSP and model checker PAT.

\section{A. RTPS StatefulWriter Module}

RTPS StatefulWriter Module has seven components. There are Publisher, DDSWriter, RTPSWriter, HistoryCache, Subscriber, DDSReader and RTPSReader. Fig. 1 shows the 22 communications in the module. It can be divided into four submodules, which are writing data, heartbeat mechanism, reading data and removing data. Here we combine all of them in Fig. 1. The detailed messages are as follows.

Writing data submodule contains the first six interactions. Publisher writes data by invoking the write operation on 


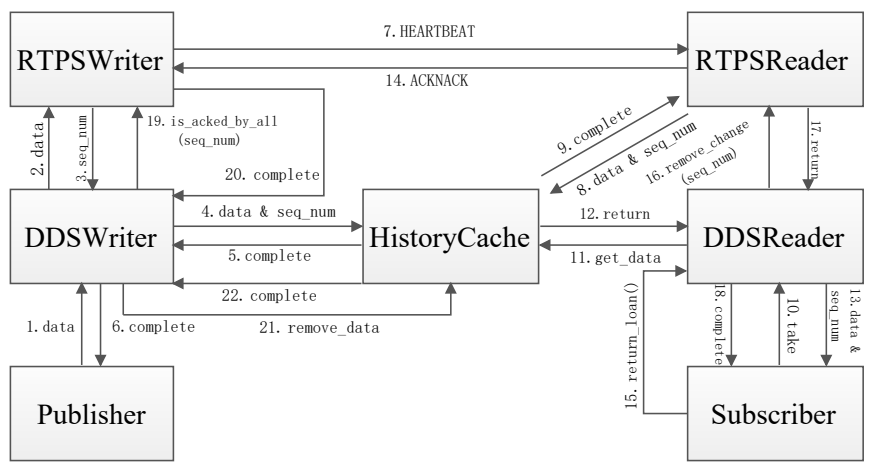

Fig. 1. Communications of RTPS StatefulWriter Module

DDSWriter. Then, DDSWriter invokes the new_change operation on RTPSWriter to create a new CacheChange. Each CacheChange has a unique sequence number. Also, DDSWriter uses the add_change operation to store the CacheChange into RTPSWriter's HistoryCache. When functions are invoked, they return the message that means operation has been executed successfully.

HeartBeat mechanism submodule is used to send message heartbeat to Reader endpoint. If the message is received smoothly within the specified time and checked by the Subscribers, RTPSWriter receives the information ACKNACK indicating confirmation.

Reading data submodule consists of four interactions. Subscriber reads data by invoking the take operation in DDSReader. Then, DDSReader accesses the changes with data and sequence number from HistoryCache. Ultimately, the take operation returns the data and sequence number to Subscriber.

Removing data submodule is composed of the remaining communications. Subscriber invokes the return_loan operation on DDSReader to notify that it no longer uses the data. Next, DDSReader uses the remove_change operation to remove the data from HistoryCache. Then, DDSWriter invokes the is_acked_by_all operation to determine whether all the changes are all received by the Reader endpoints. At length, DDSWriter calls the remove_change operation to remove the data from HistoryCache.

\section{B. A Brief Introduction to CSP and PAT}

CSP [3], [4] is a process algebra proposed by Hoare in 1978. As one of the most mature formal methods, it is tailored for describing the interaction between concurrent systems by mathematical theories. For its well-known expressive ability, CSP has been widely used in many fields [6], [8], [9]. CSP processes are constituted by primitive processes and actions. We use the following syntax to define the processes in this paper, whereby $P$ and $Q$ represent processes, $a$ and $b$ denote the atomic actions and $c$ stands for the name of a channel.

$$
\begin{gathered}
P, Q=\text { Skip } \mid \text { Stop }|a \rightarrow P| c ? x \rightarrow P|c ! e \rightarrow P| \\
P \square Q|P\|Q|P|\| Q| P ; Q \mid P[|X|] Q
\end{gathered}
$$

where:
- Skip stands for a process which only terminates successfully.

- Stop represents that the process does nothing and its state is deadlock.

- $a \rightarrow P$ first performs action $a$, then behaves like $P$.

- $c ? x \rightarrow P$ receives a message by channel $c$ and assigns it to variable $x$, then behaves like $P$.

- $c ! e \rightarrow P$ sends a message $e$ through channel $c$, then performs $P$.

- $P \square Q$ acts like either $P$ or $Q$ and the environment decides the selection.

- $P \| Q$ shows the parallel composition between $P$ and $Q$. The $\|$ means that actions in the alphabet of both operands require simultaneous participation of them.

- $P ; Q$ executes $P$ and $Q$ sequentially.

- $P[|X|] Q$ indicates that processes $P$ and $Q$ perform the concurrent events on the set $X$ of channels.

PAT Analysis Toolkit (PAT), is designed as an extensible and modularized framework for automatic system analysis based on CSP. It supports specifying and verifying systems in many different modeling languages and there are already various systems such as concurrent real-time systems, probalistic systems, activity recognition and in other domains that have been verified in PAT. PAT can be applied in verifying various properties such as divergencefree, reachability and LTL propertites with assertions in distributed systems. Here we list some notations as below.

- \#define $N$ defines a global constant $N$ with the initial value 0 .

- channel c 1 stands for a channel which has the name $c$ and the buffer size 1 .

- var cond = false represents a boolean condition with the initial value false.

- [cond $P$ indicates a guarded process, which only executes when its guard condition is satisfied.

- \#define goal $n>0$; \#assert $P$ reaches goal; defines an assertion that checks whether process $P$ can reach a state where the condition goal is satisfied.

- \#assert $P() \mid=F$; defines an assertion that checks whether process $P$ satisfies the formula $F$.

\section{Modeling RTPS StatefulWriter Module}

In this section, we give the formal model of RTPS StatefulWriter Module. The formalization is proceeded based on the communications in Fig. 1. Our model is constituted by seven core components: Publisher, DDSWriter, RTPSWriter, HistoryCache, Subscriber, DDSReader and RTPSReader.

\section{A. Sets, Messages and Channels}

Fig. 2 gives the channels of communication in the module. For more convenience, we give the definitions of sets used in 
the model. We define the set of Publisher of Publisher component, DDSWriter of DDSWriter component, RTPSWriter of RTPSWriter component, HistoryCache of HistoryCache component, DDSReader of DDSReader component and RTPSReader of RTPSReader component. In addition, we define the set: REQ of request, SEQ of sequence number messages and DATA of data information; for simplicity, ALLSETS defines the unions of all sets of RTPS StatefulWriter module.

Based on the sets defined above, the messages transmitted among components are defined as follows:

$$
\begin{aligned}
& M S G=M S G_{r e q} \cup M S G_{r e p} \cup M S G_{\text {data }} \\
& M S G_{r e q}=\left\{\text { msg }_{\text {req }} \cdot \text { A.B.content } \mid A \in(\text { ALLSETS-Publisher }),\right. \\
& B \in A L L S E T S, \text { content } \in R E Q\} \\
& M S G_{r e p}=\left\{\text { msg }_{\text {rep }} . \text { A.B.content } \mid A \in\right. \text { ALLSETS, } \\
& B \in A L L S E T S \text {, content } \in S E Q \cup R E Q\} \\
& M S G_{\text {data }}=\left\{\text { msg }_{\text {data }} \cdot A \cdot \text { B.content } \mid A \in\right. \text { ALLSETS, } \\
& B \in \text { ALLSETS, content } \in \text { DATA }\}
\end{aligned}
$$

where, $M S G_{r e q}$ represents the set of request messages, $M S G_{r e p}$ stands for the set of all kinds of response requests and $M S G_{d a t a}$ represents the set of messages transmitting data. Each message contains a tag from the set $\left\{m s g_{r e q}, m s g_{r e p}\right.$, $\left.m s g_{\text {data }}\right\}$.

Then, we give the definitions of channels. In this paper, the channels using COM_PATH to represent can be defined as follows:

$$
\begin{aligned}
& \text { ComPW, ComWP, ComWR, ComRW, ComWC, } \\
& \text { Com CW, ComCT, ComTC, ComRT, ComTR, } \\
& \text { ComCD, ComDC, ComsD, ComDS, ComDT, ComTD }
\end{aligned}
$$

The declarations of the channels are as follows:

\section{Channel COM_PATH : MSG}

Table I shows the meanings and functionalities of representative messages transferred in the channels.

TABLE I

THE EXPLANATIONS OF TYPICAL MESSAGES OF THE MODEL

\begin{tabular}{cc}
\hline Messages & Functionalities \\
\hline data, DATA & data transferred in the module \\
seq_num, SEQ_NUM & sequence number \\
heartbeat & judge whether data is received within required time \\
noinvoke & judge whether invoke functions \\
complete & judge whether execute the function \\
take & read data from cache \\
remove & remove data from cache \\
get_change & get changes from cache \\
\hline
\end{tabular}

\section{B. Overall Modelling}

System process is composed of all seven subprocesses running in parallel through their own corresponding channel. The subprocesses are Publisher, DDSWriter, RTPSWriter, HistoryCache, Subscriber, DDSReader and RTPSReader. The behavior of System process is modelled as below.

System $=_{d f}$ Publisher $\|$ DDSWriter $\|$ RTPSWriter $\|$ HistoryCache || Subscriber || DDSReader || RTPSReader

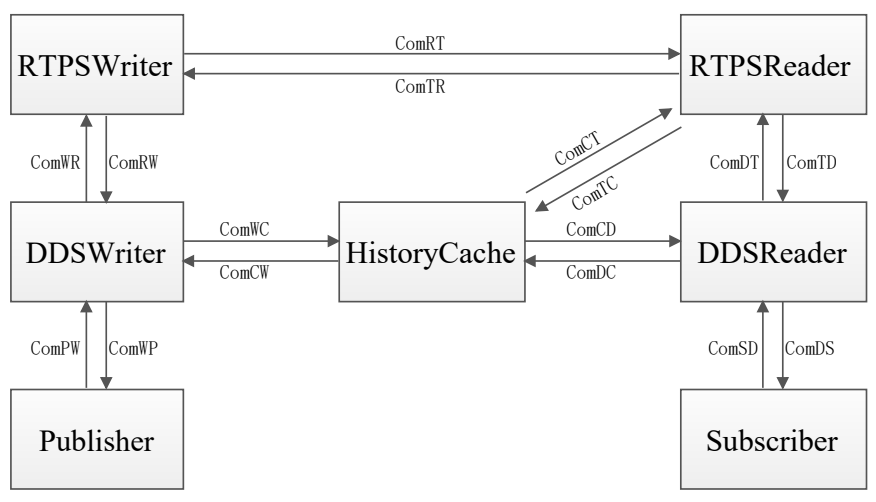

Fig. 2. Channels of RTPS StatefulWriter Module

\section{Publisher}

Publisher process is the core part in writing data submodule. It is used to write data to HistoryCache and receives the complete information from DDSWriter. The behavior of Publisher process is modelled as below.

$$
\begin{aligned}
\text { Publisher }()= & { }_{d f} \text { ComPW!msg } g_{\text {data }} . P . W . \text { data } \\
& \rightarrow \text { ComWP?msg } g_{\text {rep }} \text {.W.P.complete } \rightarrow \text { Publisher }()
\end{aligned}
$$

\section{DDSWriter}

DDSWriter process plays an important role in writing data and removing data submodule. First, it sends and receives messages to write data from Publisher. Then, it applies the acknowldgement mechanism to check if the data has been totally received. If the return message is $A C K$, it invokes function to remove data and sequence numbers from HistoryCache. The behavior of DDSWriter process is modelled as below.

$$
\begin{aligned}
& \text { DDSWriter }()={ }_{d f} \text { ComPW?msg }{ }_{\text {data }} \text {.P.W.data } \\
& \rightarrow \text { ComDR!msg }{ }_{\text {req }} \text {.W.R.data } \\
& \rightarrow \text { GetSeqNum();ComRD?msg }{ }_{\text {rep }} \text {.R.D.complete } \\
& \rightarrow \text { ComWC!ms } g_{\text {req }} \text { W.C.data.seq_num } \\
& \rightarrow \text { ComCW? msg }{ }_{\text {rep }} \text {.C.W.complete } \\
& \rightarrow \text { ComWP! } \mathrm{msg}_{\text {rep }} \text {.W.P.complete } \\
& \rightarrow \text { if (id_acked_by_all(seq_num) }==\text { true })\{ \\
& \text { ComRD?msg }{ }_{\text {rep }} \text {.R.D.complete } \\
& \rightarrow \text { ComWC!msg } g_{r e q} \text {.W.C.remove } \\
& \rightarrow \text { remove_change(seq_num); } \\
& \text { ComCW?msg }{ }_{\text {rep }} \text {.C.W.complete } \\
& \rightarrow \text { DDSWriter }()\} \text { else }\{\text { Skip }\}
\end{aligned}
$$

In the above formula, GetSeqNum() is used to set the number of the sequence; id_acked_by_all(seq_num) is a function that judges whether the data with the seq_num is acknowledged; remove_change(seq_num) is used to remove the changes in the HistoryCache component.

\section{E. RTPSWriter}

RTPSWriter process works in writing data and heartbeat mechanism submodule. First, it produces the unique sequence number for the uploaded data. Second, it uses heartbeat mechanism to send heartbeat to RTPSReader for assuring the data can be transferred within the required interval. Finally, it helps to check whether the sequence numbers are checked by 
all Subscribers. The behavior of RTPSWriter is modelled as below.

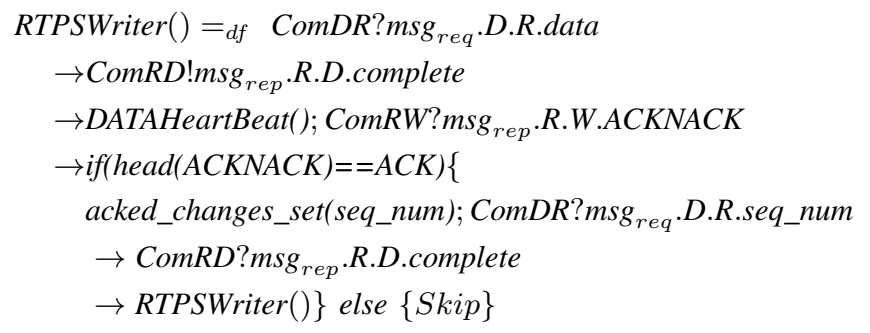

ACKNACK contains $A C K$ and seq_num, so we use head(ACKNACK) to retrieve the message $A C K$. acked_changes_set(seq_num) checks whether the changes are set. HeartBeat mechanism is very important in the model. Its detailed behavior is modelled as follows:

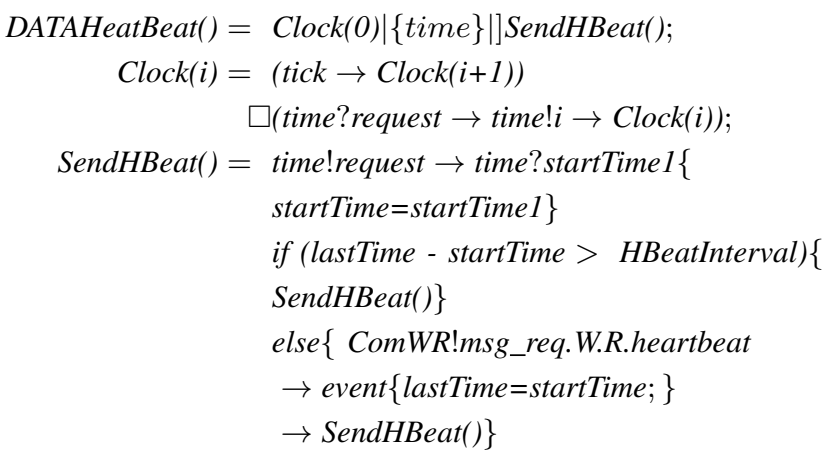

time is the channel between Clock and SendHBeat(); Clock $(i)$ process returns the current time if receives the request message. SendHBeat() process sends the heartbeat message if the time difference is less than HBeatInterval; otherwise, the process cycle continues.

\section{F. HistoryCache}

HistoryCache process is like a database mainly for storing data and corresponding sequence number. It functions in every submodule, such as writing data and removing data. When receiving the request from Publishers or Subscribers, it invokes the homologous function to handle. The behavior of HistoryCache process is modelled as below.

$$
\begin{aligned}
& \text { HistoryCache }()=d f \text { ComWC?msg }{ }_{r e q} . \text { W.C.data.seq_num } \\
& \rightarrow \text { ComCW!msg } g_{\text {rep }} \text {.C.W.complete } \\
& \rightarrow \text { ComTC?msg }{ }_{\text {req. }} \text { T.C.data.seq_num } \\
& \rightarrow \text { ComCT!msg } g_{\text {rep }} \text {.C.T.complete } \\
& \rightarrow \text { complete12:=get_changes(seq_num); } \\
& \text { ComCR!msg }{ }_{\text {rep }} \text {.C.R.complete12 } \\
& \rightarrow \text { ComWC?msg } g_{\text {req. W.C.remove_change }} \\
& \rightarrow \text { complete23:=remove_changes(seq_num); } \\
& \text { ComCW!msg rep.C.W.complete23 } \\
& \rightarrow \text { HistoryCache }()
\end{aligned}
$$

In the above formula, function get_changes(seq_num) and remove_changes(seq_num) is used to get and remove changes from HistoryCache component, respectively. Both of them can return the value 1 to indicate the operation is successful; otherwise, they return 0 .

\section{G. Subscriber}

Subscriber process is designed for reading data and removing data submodule. First of all, it calls take function to receive data from HistoryCache. Next, it notifies other components that the data will not be used and gets the corresponding feedback. The behavior of Subscriber process is modelled as below.

$$
\begin{aligned}
& \text { Subscriber }()={ }_{d f} \text { ComSR!msg } g_{r e q} \text {.S.R.take } \\
& \rightarrow D A T A:=\text { take(); ComRS?msg }{ }_{r e p} . \text { R.S.DATA } \\
& \rightarrow \text { ComSR!msg }{ }_{\text {req }} \text {.S.R.loan } \rightarrow \text { noinvoke }:=\text { return_loan(); } \\
& \text { ComRS?msg } g_{\text {rep }} \text {.R.S.noinvoke } \rightarrow \text { Subscriber() }
\end{aligned}
$$

In the above formula, function take() reads data from HistoryCache component and return_loan() indicates the data is not invoked any more, whose value is assigned to noinvoke.

\section{H. DDSReader}

DDSReader process is used for reading data and removing data submodule. First, it helps the Subscriber get data and sequence number from HistoryCache. Then, it invokes remove_change function to remove changes in HistoryCache. The behavior of DDSReader is modelled as below.

$$
\begin{aligned}
& \text { DDSReader }()={ }_{d f} \text { ComSR?msg } g_{\text {req }} \text { S.R.take } \\
& \rightarrow \text { ComRC!msg } g_{r e q} \text {.R.C.get_change } \rightarrow \text { ComCR? } \text { msg }_{\text {rep }} \text {.C.R.complete } \\
& \rightarrow \text { ComRS!msg }{ }_{\text {rep }} \text {.R.S.DATA } \rightarrow \text { ComSR? } \text { msg }_{r e q} \text {.S.R.loan } \\
& \rightarrow \text { ComRS!ms } g_{\text {rep }} \text {.R.S.noinvoke } \rightarrow \text { ComDT!msg } g_{\text {req }} \text {.D.T.remove } \\
& \rightarrow \text { noinvoke } 2:=\text { remove_changes();ComTD? } \mathrm{msg}_{\text {rep }} \text {.T.D.noinvoke } 2 \\
& \rightarrow \text { DDSReader }()
\end{aligned}
$$

In the above formula, function remove_changes() is the same as that in process HistoryCache. take and get_change are the messages to invoke take() and get_changes() function, respectively; loan and remove message are to invoke return_loan() and remove_changes() function, respectively.

\section{RTPSReader}

RTPSReader process is applied to hearbeat mechanism and removing data submodule. First, it receives the heartbeat from RTPSWriter and sends the timely feedback to RTPSWriter. Then, it assists the Subscriber to remove the changes and data in HistoryCache. The behavior of RTPSReader is modelled as below.

$$
\begin{aligned}
& \text { RTPSReader }()={ }_{d f} \text { ComWR? } \mathrm{msg}_{\text {req }} . \text { W.R.heartbeat } \\
& \rightarrow \text { ComTC!msg } g_{\text {req.T.C.data.seq_num } \rightarrow \text { ComCT?msg }} \text { rep.C.T.complete } \\
& \rightarrow \text { ComRW!msg }{ }_{\text {rep }} \text {.R.W.ACKNACK } \rightarrow \text { ComDT?msg } g_{\text {req }} \text {.D.T.remove } \\
& \rightarrow \text { ComTD!msg }{ }_{\text {rep }} \text {.T.D.noinvoke } 2 \rightarrow \text { RTPSReader }()
\end{aligned}
$$

In the above formula, RTPSReader receives heartbeat, sends data and seq_num and most importantly, sends ACKNACK to complete the procedure of the acknowledgement mechanism.

\section{IMPLEMENTATION AND VERIFICATION}

In this section, the model in Section III is implemented in the model checker PAT and the properties abstracted from the specification are all verified. 


\section{A. Implementation}

First, we need to define important channels, message type flags and delivery objects as enumerations, and define messages communicated between channels as global variables. For the definition of the above variables, we give the following list as a reference:

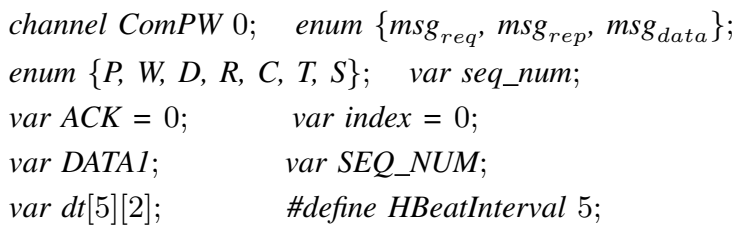

All other channels in the model are defined by the above channel format syntax like $\operatorname{ComPW}$; the enumerated types are the type of the flag message, including $m s g_{r e q}$ to represent the request, $m s g_{r e p}$ to stand for the reply, and $m s g_{\text {data }}$ to represent the data; $P, W, D, R, C, T, S$ represent the English capital initials of the seven modules in the RPTS StatefulWriter module model section. Global variable $A C K$ initialized to 0 means no data received is checked by the Subscriber; global variable index initialized to 0 means the number of the data stored in the array in HistoryCache. seq_num means the initialized sequence number is zero; $D A T A 1$ and $S E Q \_N U M$ are the variable representing data and sequence number in Subscriber component. Array $d t[5][2]$ stores data and corresponding suquence number in HistoryCache component. Also, we give the definitions of some constant variables, for example, HBeatInterval, whose manual value is set to 5 .

Then, we give the code of one of the processes in PAT as an example. Here we take the implementation of the DDSWriter() process as an example:

$$
\begin{aligned}
& \text { DDSWriter }()=\text { ComPW?msg_data.P.W.datal }\{\text { data }=\text { datal }\} \\
& \rightarrow \text { ComWR!msg_req.W.R.data } \rightarrow \text { GetSeqNum(); } \\
& \text { ComRW?msg_rep.R.W.complete } 3 \text { complete }=\text { complete } 3\} \\
& \rightarrow \text { ComWC!msg_req.W.C.data.seq_num } \\
& \rightarrow \text { ComCW?msg_rep.C.W.complete } 5\{\text { complete }=\text { complete } 5\} \\
& \rightarrow \text { ComWP!msg_rep.W.P.complete } \\
& \rightarrow \text { ComWR!msg_req.W.R.is_acked_all } \\
& \rightarrow \text { if }(\text { call }(\text { is_acked_by_all,seq_num })==1)\{ \\
& \text { ComRW?msg_rep.R.W.complete } 21\{\text { complete }=\text { complete } 21\} \\
& \rightarrow \text { ComWC!msg_req.W.C.remove } \\
& \rightarrow \text { Remove()\} else }\{\text { Skip }\} \text {; }
\end{aligned}
$$

From the above process execution code, it can be seen that datal event assigns variables and ensures variable values of all processes in the entire system are consistently changed. The function is_acked_by_all is invoked by call. GetSeqNum() and Remove() are other processes used to enhance the readability. Apparently, GetSeqNum() is used to get the sequence number; Remove() is used to remove the changes from the HistoryCache component. Their details are as follows.

$$
\begin{aligned}
\operatorname{GetSeqNum}()= & \text { getSeqNum }\{ \\
& \text { seq_num }=\text { seq_num }+1 ;\} \rightarrow \text { Skip } ;
\end{aligned}
$$

If GetSeqNum() is executed once, seq_num pluses 1, which can keep the sequence number always different and unique.

$$
\begin{aligned}
\text { Remove }()= & \text { atomic }\{ \\
& i f(\text { call }(\text { remove_change,seq_num })==1)\{ \\
& \text { ComCW?msg_rep.C.W.complete } 23\{ \\
& \text { complete }=\text { complete } 23\} \rightarrow \text { Skip }\} \\
& \text { else }\{\text { ComCW } ? \text { msg_rep.C.W.nocomplete } 23\{ \\
& \text { complete }=\text { complete- } 1 ; \\
& \text { complete }=\text { nocomplete } 23\} \rightarrow \text { Skip }\} ;
\end{aligned}
$$

We use atomic to define Remove() process, which means that the event cannot be disturbed until it is finished. complete 23 and nocomplete 23 are the event used to transimitting corresponding complete meassge.

Finally, the full definition of the entire system is given as follows:

$$
\begin{aligned}
\operatorname{SYSTEM}()= & \text { Publisher() } \| \text { DDSWriter() } \| \\
& \text { RTPSWriter() } \| \text { HistoryCache() } \| \\
& \text { Subscriber() } \| \text { DDSReader() } \| \text { RTPSReader(); }
\end{aligned}
$$

\section{B. Properties Verification}

Based on the implementation of the model in PAT above, we verify four properties as follows:

\section{1) Divergence free}

$$
\text { \#assert System() divergencefree; }
$$

Divergence free means that any traces of the system can diverge rather than behave chaotically.

\section{2) Consistency}

Property data consistency is so important that the data from Publisher or HistoryCache or Subscriber component must be completely identical. In the implementation, the original value of the transferred data is equal to 2 and its corresponding sequence number should be equal to 1 . If the data and sequence number are consistent in different components, the property is satisfied. Thus, we give the definition and assertion as follows:

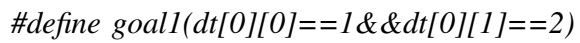

$$
\begin{aligned}
& \& \&\left(D A T A 1==2 \& \& S E Q \_N U M==1\right) \\
& \& \&\left(d t[0][0]==S E Q \_N U M \& \& d t[0][1]==D A T A 1\right) \text {; } \\
& \text { \#assert SYSTEM() reaches goal1; }
\end{aligned}
$$

\section{3) Acknowledgement Mechanism}

The reliable pattern has an acknowledgement mechanism. In our model, if the final value of the global variable $A C K$ and index are all changed from 0 to 1 , the property is satisfied. Thus, we give the LTL formula and reachability to verify whether the property is safe. Their definitions and assertions are as follows:

$$
\begin{aligned}
& \text { \#define goal2 }(A C K==1 \& \& \text { index }==1) \text {; } \\
& \text { \#assert SYSTEM() reaches goal } 2 \\
& \text { \#assert SYSTEM }() \mid=<>\text { goal } 2
\end{aligned}
$$

\section{4) Sequentiality}

If the Publisher component sends several pieces of data in sequence, the pattern needs to guarantee that the data stored in the HistoryCache component must be in order. Thus, we give three atomic processes SYSTEM02(), SYSTEM03() 
and SYSTEM04() on the basis of process SYSTEM(). If their storage order is correct in HistoryCache, the property is satisfied. Their definitions and assertions are as follows:

$$
\begin{aligned}
& \text { SYSTEM02( }()=\text { atomic }\{\text { event }\{\text { data }=2 ;\} \rightarrow \operatorname{SYSTEM}()\} ; \\
& \text { SYSTEM03() }=\text { atomic }\{\text { event }\{\text { data }=4 ;\} \rightarrow \operatorname{SYSTEM}()\} ; \\
& \text { SYSTEM04( })=\text { atomic }\{\text { event }\{\text { data }=6 ;\} \rightarrow \operatorname{SYSTEM}()\} \text {; } \\
& \text { SYSTEM05() = SYSTEM04()||SYSTEM03()||SYSTEM02(); } \\
& \text { \#define goal3 }(d t[0][1]==2 \& \& d t[1][1]==4 \& \& d t[2][1]==6) \text {; } \\
& \text { \#assert SYSTEM05() reaches goal3; }
\end{aligned}
$$

\section{Verification and Results}

According to the definitions and assertions, we implement the code in PAT and as a result, Fig. 3 shows the properties are all valid, which means the pattern of the module with no intruders is exactly reliable and also caters for the specification.

\section{CONClusion AND Future WORK}

RTPS StatefulWriter module is a vital component in RTPS protocol. This paper has formalized seven components comprising the Publisher, DDSWriter, RTPSWriter, DDSWriter, HistoryCache, Subscriber, DDSReader and RTPSReader with CSP. Our work also has applied the model checker PAT to implement the constructed model. Four properties abstracted from the specification, including divergence free, acknowledgement mechanism, data consistency and sequentiality, have been verified. The results are all valid. Consequently, we conclude that from the perspective of process algebra, the constructed model meets these properties and the pattern is absolutely reliable and caters for the specification.

It is naturally a great challenge to model and verify the whole RTPS protocol. We will explore security analysis and verification of the module by adding intruders in the future.

\section{ACKNOWLEDGEMNET}

This work was partly supported by National Natural Science Foundation of China (Grant No. 61872145), Shanghai Collaborative Innovation Center of Trustworthy Software for Internet of Things (No.ZF1213) and Special Fund for International Academic Conferences of Graduate Students in East China Normal University.

\section{REFERENCES}

[1] Alaerjan, A., Kim, D., Kafaf, D.A.: Modeling functional behaviors of DDS. In: 2017 IEEE SmartWorld, Ubiquitous Intelligence \& Computing, Advanced \& Trusted Computed, Scalable Computing \& Communications, Cloud \& Big Data Computing, Internet of People and Smart City Innovation, SmartWorld/SCALCOM/UIC/ATC/CBDCom/IOP/SCI 2017, San Francisco, CA, USA, August 4-8, 2017. pp. 1-7 (2017)

[2] Beckman, K., Reininger, J.: Adaptation of the DDS security standard for resource-constrained sensor networks. In: 13th IEEE International Symposium on Industrial Embedded Systems, SIES 2018, Graz, Austria, June 6-8, 2018. pp. 1-4 (2018)

[3] Brookes, S.D., Hoare, C.A.R., Roscoe, A.W.: A theory of communicating sequential processes. J. ACM 31(3), 560-599 (1984)

[4] Hoare, C.A.R.: Communicating Sequential Processes. Prentice-Hall (1985)

[5] Liu, Y., Guan, Y., Li, X., Wang, R., Zhang, J.: Formal analysis and verification of DDS in ROS2. In: 16th ACM/IEEE International Conference on Formal Methods and Models for System Design, MEMOCODE 2018, Beijing, China, October 15-18, 2018. pp. 62-66 (2018)

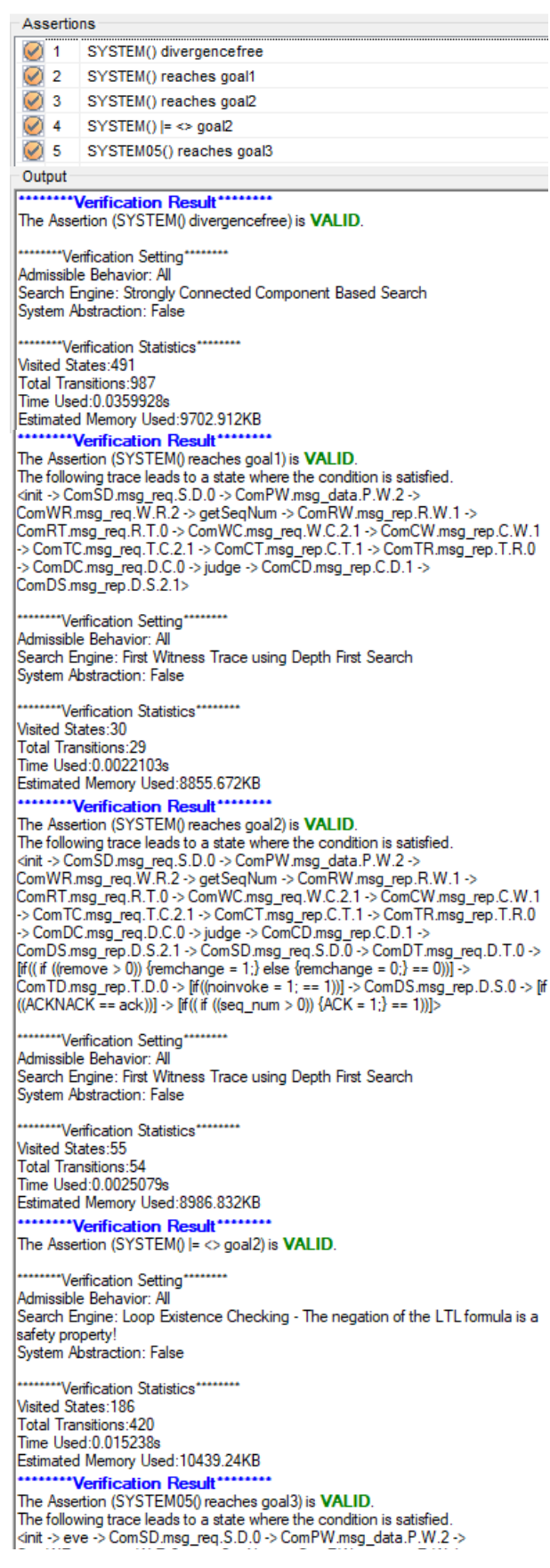

Fig. 3. Verification Result

[6] Lowe, G., Roscoe, A.W.: Using CSP to detect errors in the TMN protocol. IEEE Trans. Software Eng. 23(10), 659-669 (1997)

[7] Pérez, H., Gutiérrez, J.J.: Modeling the qos parameters of DDS for eventdriven real-time applications. Journal of Systems and Software 104, 126140 (2015)

[8] Roscoe, A.W.: The Theory and Practice of Concurrency. Prentice Hall (1997)

[9] Roscoe, A.W.: Understanding Concurrent Systems. Texts in Computer Science, Springer (2010)

[10] Youssef, T.A., Hariri, M.E., Elsayed, A.T., Mohammed, O.A.: A ddsbased energy management framework for small microgrid operation and control. IEEE Trans. Industrial Informatics 14(3), 958-968 (2018) 